\title{
New perspectives in amblyopia therapy on adults: a critical role for the excitatory/inhibitory balance
}

\author{
Laura Baroncelli ${ }^{1}$, Lamberto Maffei ${ }^{1,2}$ and Alessandro Sale ${ }^{1 *}$ \\ 1 Institute of Neuroscience, National Research Council, Pisa, Italy \\ ${ }^{2}$ Laboratory of Neurobiology, Scuola Normale Superiore, Pisa, Italy
}

\author{
Edited by: \\ Yehezkel Ben-Ari, Institut National de \\ la Santé et de la Recherche Médicale, \\ France \\ Reviewed by: \\ Enrico Cherubini, International School \\ for Advanced Studies, Italy \\ Corette Wierenga, Max Planck \\ Institute of Neurobiology, Germany \\ *Correspondence: \\ Alessandro Sale, Institute of \\ Neuroscience, National Research \\ Council, Via Moruzzi 1, I-56124 Pisa, \\ Italy. \\ e-mail:sale@in.cnr.it
}

Amblyopia is the most common form of impairment of visual function affecting one eye, with a prevalence of about $1-5 \%$ of the total world population. This pathology is caused by early abnormal visual experience with a functional imbalance between the two eyes owing to anisometropia, strabismus, or congenital cataract, resulting in a dramatic loss of visual acuity in an apparently healthy eye and various other perceptual abnormalities, including deficits in contrast sensitivity and in stereopsis. It is currently accepted that, due to a lack of sufficient plasticity within the brain, amblyopia is untreatable in adulthood. However, recent results obtained both in clinical trials and in animal models have challenged this traditional view, unmasking a previously unsuspected potential for promoting recovery after the end of the critical period for visual cortex plasticity. These studies point toward the intracortical inhibitory transmission as a crucial brake for therapeutic rehabilitation and recovery from amblyopia in the adult brain.

Keywords: amblyopia, neural plasticity, environmental enrichment, fluoxetine, perceptual learning, GABAergic inhibition
The development of brain circuitry is the result of a complex interaction between genetic programs defining the gross cerebral architecture and activity-driven processes of synaptic fine tuning. In the visual system of primates, a significant portion of neuronal pathways' maturation is accomplished during prenatal development, allowing vision to start at birth. Initially, however, visual function abilities are still highly immature and they undertake strong improvements during the successive months of life, when sensory experience exerts a dramatic influence in driving the subtle wiring of neural circuitries (Weliky, 2000; Lewis and Maurer, 2009).

The essential role of experience is particularly evident within restricted time windows in early postnatal life, the so-called critical periods (CPs; Berardi et al., 2000), during which brain circuits display a high sensitivity to acquire instructive and adaptive signals from the external environment. Accordingly, early disruption of proper environmental inputs caused by conditions of visual deprivation or ocular abnormalities can result in long-term or even permanent brain diseases (Brémond-Gignac et al., 2011). Among them, amblyopia (lazy eye) is a severe disorder that, aside from refractive error, is the most common cause of vision loss during infancy, with an estimated prevalence of $1-5 \%$ in the population (Holmes and Clarke, 2006). Amblyopia emerges from untreated conditions of early abnormal visual experience in which a functional imbalance between the two eyes is predominant owing to anisometropia (unequal refractive power in the two eyes), strabismus (abnormal alignment of ocular axes with each other), or congenital cataract (clouding in the crystalline lens obstructing light transmission; Mittelman, 2003).

Much of our current understanding of the neural mechanisms underlying this disorder derives from studies on animal models, revealing that the major pathological changes in amblyopia occur at the cortical level. The seminal work performed by Hubel and Wiesel in kittens showed that reducing input from one eye by lid suture, a treatment usually referred to as monocular deprivation (MD), severely affects the physiology and anatomy of the visual cortex, with a delayed development of visual acuity and contrast sensitivity for the deprived-eye accompanied by disruption of cortical binocularity properties (Wiesel and Hubel, 1963). Similarly, amblyopia in humans is characterized by a dysfunction of sensory information neural processing, leading to a dramatic degradation of visual acuity in absence of structural abnormalities at the ocular examination and despite appropriate optical correction (Holmes and Clarke, 2006). In addition, the clinical picture of amblyopia is frequently complicated by the presence of a broad range of other perceptual deficits, including contrast sensitivity and stereopsis defects (Kiorpes, 2006; Levi, 2006).

The prevailing consensus is that amblyopia reversal is only possible early in life, before the closure of CP. Accordingly, precocious diagnosis and correction of any visual deprivation source is crucial for preventing visual impairments to become permanent (Holmes and Clarke, 2006). The traditional amblyopia therapy consists in patching or penalizing the fellow preferred eye, thus forcing the brain to use the visual input carried by the amblyopic eye (Wu and Hunter, 2006). The success rate of this treatment is dependent on several factors, including seriousness of visual ability disruption, type of amblyopia, occlusion dose, patient compliance, and age of onset (Stewart et al., 2005).

Despite the dogma that amblyopia is an untreatable pathology in adults, recent studies on animal models and clinical trials have challenged this picture, providing exciting evidence that intervention strategies boosting brain plasticity in adulthood may allow 
the reinstatement of visual functions in amblyopic subjects well after the end of the CP.

\section{RECOVERY FROM LONG-TERM VISUAL DEPRIVATION: LESSONS FROM ANIMAL MODELS}

Significant effort is being made in multiple laboratories to elaborate new intervention procedures aimed at inducing juvenile-like neural plasticity in the adult brain. In addition to the theoretical relevance of these studies in a basis research perspective, they may have a great impact also on clinical practice: promoting plasticity in the adult nervous system, indeed, could pave the way for the development of innovative therapies for brain disorders for which a suitable treatment is still not available in adulthood (Bavelier et al., 2010).

In animal models, the amblyopic condition can be induced by long-term MD starting during the $\mathrm{CP}$ and protracted until adulthood. New experimental protocols successfully employed as strategies for enhancing adult brain plasticity can be classified in two categories on the basis of the general approach followed (Figure 1).

On the one hand, pharmacological manipulations of functional and structural brakes limiting plasticity to the CP have been shown to restore normal visual functions in adult amblyopic animals. Several studies pointed to intracortical inhibition as a key factor for defining the boundaries of plasticity, suggesting that a reduction of transmission in interneurons that release GABA $(\gamma$ aminobutyric acid) could be a crucial step for the restoration of plasticity processes in adulthood (for review, see Hensch, 2005 and Baroncelli et al., 2011).

The most direct demonstration that inhibitory transmission limits plasticity in the adult brain derives from a recent study reporting that pharmacological reduction of intracortical inhibition is sufficient to reopen a window of plasticity in the visual cortex well after the closure of the CP (Harauzov et al., 2010). First studies in animal models of amblyopia reported that the administration of anti-inhibitory compounds leads to a substantial restoration of the input from the deprived eye to the visual cortex (Duffy et al., 1976). Despite its theoretical appealing, reducing inhibition levels with direct pharmacological treatments can raise concerns about the effective clinical value, since some GABAergic transmission antagonists are of very limited utility for their pro-convulsive action, while others have not been approved by the FDA.

Brainstem neuromodulatory systems, such as those containing noradrenalin, serotonin, and acetylcholine, project to the cortex targeting GABAergic interneuron and specifically affecting the output of these cells (Bacci et al., 2005). Thus, an alternative way for adjusting the balance between excitatory and inhibitory transmission to levels favorable for plasticity may be an artificial regulation of the endogenous release of these transmitters. About 30 years ago, it has been reported that an increase in the local availability of noradrenalin enhances neuronal plasticity, accelerating cortical recovery from the effects of prior MD (Kasamatsu, 1982). In agreement with this previous finding, we recently demonstrated that chronic administration of fluoxetine, a selective serotonin reuptake inhibitor enhancing extracellular serotonin and noradrenalin levels, reactivates cortical plasticity in adulthood promoting a full recovery of visual functions in amblyopic animals through a reduction of inhibitory transmission (Maya Vetencourt et al., 2008). Treating amblyopia with fluoxetine is a promising approach if one considers that this substance is a FDA-approved drug widely prescribed in the treatment of depression and for which a very good knowledge of both beneficial and side effects is available. Interestingly, the permissive action of neuromodulatory transmitters on brain plasticity is developmentally regulated by increased levels of molecules that limit cerebral circuit reorganization in adulthood. It has been shown, indeed, that the expression of Lynx1, an endogenous prototoxin which directly binds to nicotinic receptors reducing their sensitivity to acetylcholine, increases after the closure of the CP and that the genetic removal of the molecular brake provided by this protein restores visual cortex plasticity in adult animals (Morishita et al., 2010).

Another molecular factor recently linked to the control of cortical plasticity levels is histone acetylation. Acetylation of histones $\mathrm{H} 3$ and $\mathrm{H} 4$ is developmentally down-regulated by an experiencedependent process related to the closure of the $\mathrm{CP}$ for visual cortex plasticity (Putignano et al., 2007). A pharmacological epigenetic treatment increasing histone acetylation (i.p. injection of valproic acid, VPA) emerged to be effective in adult rats in reversing visual acuity deficits induced by long-term MD (Silingardi et al., 2010). To date, no effects on the inhibitory transmission have been documented following manipulations of the epigenetic machinery. However, since prenatal exposure to inhibitors of histone deacetylases decreases the number of parvalbumin-positive inhibitory neurons in the neocortex of adult mice, it has been proposed that one mechanism of action for drugs targeting histone acetylation may be an adjustment of the excitatory-inhibitory ratio in cerebral circuitries (Gogolla et al., 2009).

Moving from the intracellular to the extracellular milieu, it has been shown that infusion in the mature cortex of amblyopic rats of an enzyme (chondroitinase ABC) that degrades chondroitin sulfate proteoglycans (CSPGs), an essential component of the brain extracellular matrix (ECM), produces a marked reinstatement of both visual acuity and binocularity. Since most CSPG-containing nets are localized around the soma of inhibitory interneurons, the permissive action of ECM degradation on cortical plasticity could occur through a direct structural and functional remodeling of inhibitory synapses (Pizzorusso et al., 2006).

On the other hand, pronounced improvements in visual functions have been obtained by experimental paradigms based on the manipulation of environmental stimulation levels. A recent study reported that exposing adult animals to complete darkness can induce vision recovery in amblyopic rats, providing evidence that the enhanced cortical plasticity is related to a shift in the balance between excitation and inhibition toward juvenile-like levels (He et al., 2007). Clinical translation of this treatment, however, remains uncertain, since a long dark exposure is likely to be disruptive for most people.

A more promising approach is environmental enrichment (EE). The goal of EE is to improve the animals' quality of life by providing them with a combination of multi-sensory/cognitive stimulation, increased physical activity and enhanced social interactions. EE is a gain-of-function paradigm allowing the study of the influence elicited by increased levels of environmental stimulation on brain 


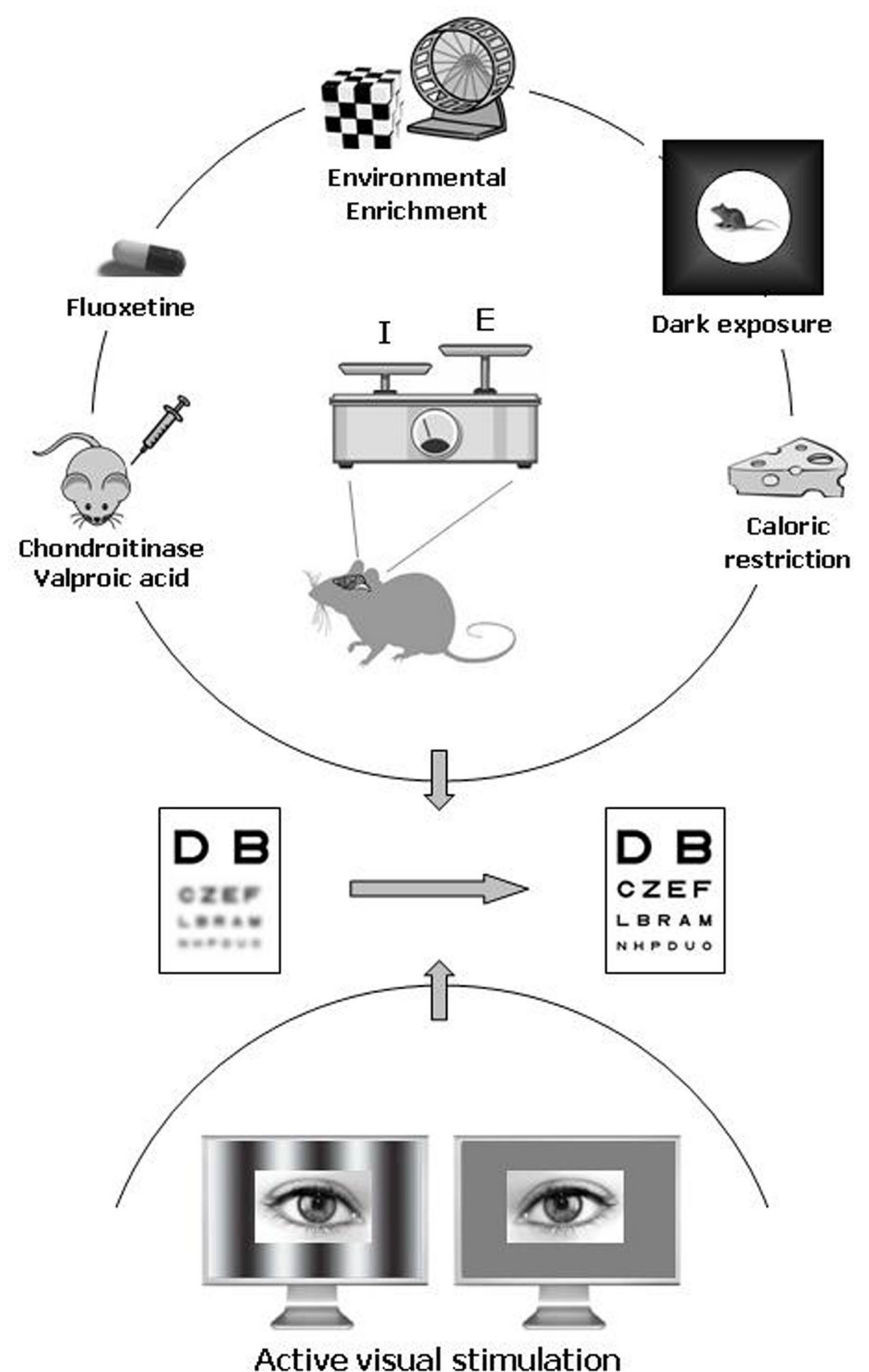

FIGURE 1 | Pharmacological and environmental therapeutic strategies for amblyopia in adulthood. Recent data have documented a previously unsuspected high potential of neuronal plasticity in the adult visual cortex. In animal models, plasticity can be elicited either by pharmacological treatment with chronic administration of antidepressants (fluoxetine), valproic acid (an inhibitor of histone deacetylases), or chondroitinase ABC (which degrades the extracellular matrix chondroitin sulfate proteoglycans), and by exposure to environmental enrichment, housing in complete darkness, or caloric restriction. In humans, emerging clinical studies point to active visual stimulation obtained with perceptual learning or playing video games as a promising strategy for treating amblyopia in adulthood. An increased ratio between excitation and inhibition owing to a reduced intracortical inhibitory tone is thought to be a central hub triggering plasticity in the adult visual cortex. plasticity (van Praag et al., 2000; Sale et al., 2009; Baroncelli et al., 2010). We showed that EE is highly effective for treating amblyopia in adulthood: a brief exposure of adult amblyopic rats to EE promotes a complete recovery of both visual acuity and ocular dominance. Recovery of plasticity in enriched animals is paralleled by a marked reduction of the visual cortex inhibitory tone, for which we demonstrated a causal role in the enhancement of plasticity induced by EE (Sale et al., 2007). 
An alternative approach for modulating physiological brain function has been shown to be the regulation of caloric intake. Nutrition is a fundamental component of the environment playing a key role in prenatal and postnatal visual development (BrémondGignac et al., 2011). It has been recently reported that a short-term protocol of food restriction starting in adulthood is able to restore neural plasticity in the visual system, renewing the capability of recovery from amblyopia in long-term deprived animals. Also in this case the effects on cortical plasticity were associated with a marked reduction of GABAergic inhibition (Spolidoro et al., 2011).

The picture emerging from this brief survey of the most recent literature in animal models is that the ratio between excitation and inhibition is a critical factor controlling the possibility to induce recovery from amblyopia in the adult. Given the morphological and functional complexity of inhibitory circuitries in the vertebrate brain, the precise role of GABAergic inhibition in limiting plasticity in the adult cortex is still under debate. Neural circuits, indeed, rely on inhibition mediated by diverse classes of interneurons with distinct morphologies, physiological properties, and subcellular innervation patterns. Moreover, GABAergic transmission fulfills multiple functions going from regulation of synaptic integration and timing of action potential generation to control of network oscillations (Huang et al., 2007). Parvalbuminpositive basket cells innervating the soma of target neurons with synapses containing the $\alpha 1$ subunit of $\mathrm{GABA}_{\mathrm{A}}$ receptors are currently considered critical for visual cortex plasticity regulation (Hensch, 2005).

It has been suggested that reducing inhibition promotes adult visual cortical plasticity by increasing the capability of the cortex to relay incoming patterns of activity to the supragranular layers (Kirkwood and Bear, 1994; Rozas et al., 2001). In agreement with this hypothesis, infusion of MPA or picrotoxin in the adult visual cortex enhances the possibility to induce activity-dependent longterm potentiation (LTP) of synaptic efficacy, but not long-term depression, both in layers II-III and IV (Harauzov et al., 2010). Thus, a reduction of GABAergic activity would favor recovery from amblyopia by facilitating a potentiation of the excitatory inputs from the undeprived eye.

Even if the prevailing consensus is that the major functional effects of vision deprivation in one eye result from plasticity at excitatory connections in the visual cortex, recent research has brought attention to the alternative possibility that intracortical inhibition of deprived-eye inputs could also increase, leading to a suppression of the visual responses evoked by the deprived eye (for review, see Smith and Bear, 2010). However, dissecting the role of plasticity at both excitatory and inhibitory synapses in the amblyopic condition deserves further investigation. It is also worth stressing that, to our knowledge, amblyopia has been never associated with a depolarizing and excitatory action of GABA, which has been instead reported for other pathological conditions, including epilepsy (Cohen et al., 2002; Huberfeld et al., 2007), neuropathic pain (Coull et al., 2005), inflammatory hyperalgesia and allodynia (Funk et al., 2008), and Alzheimer's disease (Lagostena et al., 2010).

\section{IMPACT OF ACTIVE VISUAL STIMULATION IN ADULT AMBLYOPIC HUMAN SUBJECTS}

At the clinical level, biological manipulations effective in restoring neural plasticity in the mature brain should be translated into feasible and safe interventions in order to represent a significant advance in the field of amblyopia treatment. A growing number of recent clinical studies pointed to perceptual learning (PL) as a very promising strategy for treating amblyopia in adulthood (Figure 1). PL refers to any change in perceptual ability as a result of practice and can be observed in all sensory modalities. In the visual system, practice with procedures of specific sensory enrichment improves performance in a variety of tasks, such as grating, texture, hyperacuity, or stereoscopic discrimination (for review, see Fine and Jacobs, 2002; Fahle, 2004, 2005). This form of neural plasticity does not seem to be an exclusive prerogative of a physiologically normal visual system, since it has been repeatedly observed also in adult people with amblyopia.

As early as 1970s, Campbell et al. (1978) reported that passive stimulation of the amblyopic eye with high-contrast square-wave rotating gratings of different spatial frequencies induced a substantial improvement in high-frequency contrast sensitivity and grating acuity in children. The method used in this seminal work, usually referred to as the Cambridge stimulator or CAM treatment, can be considered as a first example of a very simple PL procedure applied to the treatment of amblyopia. After a successive period of criticism in which the validity of this concept has been challenged by a number of negative results, in the last 15 years numerous papers have started to document various and robust beneficial effects on visual functions elicited by PL in adult amblyopes whose age was always higher than the 7-years cut-off classically considered the limit for a successful intervention. Importantly, no correlation between population age and functional outcome of the treatment has been ever reported in these studies (e.g., Polat et al., 2004; Chen et al., 2008). Moreover, a comparative inspection of the obtained results has allowed noticing that the age of the subjects enrolled in the various tested experimental procedures is not the main factor accounting for the variance across studies (see Levi and Li, 2009a).

While it is undisputed that PL involves changes on high cognitive levels of visual information processing, it also relies at least partly on modifications on earlier levels (Fahle, 2004). It has been reported, indeed, that PL has the ability to elicit plastic changes in the visual cortex, as shown by Yotsumoto et al. (2008) who observed a change in blood-oxygen-level dependence (BOLD) signal in human primary visual cortex (V1) following visual PL. In the same line, we recently observed that visual PL is accompanied by LTP of thalamo-cortical and cortico-cortical synaptic responses in the rat V1 (Sale et al., 2011), a direct demonstration that PL results in V1 neural plasticity. Accordingly, Cooke and Bear (2010) reported that repeated presentation of a sinusoidal grating stimulus over days induces LTP in the V1 of awake mice. Since it is currently believed that alterations in neural responses in the early visual cortex are the primary cause of vision dysfunction in amblyopia (Kiorpes, 2006; Levi, 2006), the possibility to promote V1 plasticity in a totally non-invasive manner with PL is very promising in the context of amblyopia treatment. 
On the other side, since PL also occurs at early stages of visual processing, it may show a striking selectivity for the stimulus parameters. This raises one caveat to its therapeutic value in the treatment of amblyopia, because the achievable improvements might be limited to the selected trained stimulus, condition, or task (Levi and Li, 2009b). However, differently from what found in healthy subjects, the available results reported until nowadays do not show such a narrow specificity for the trained task in amblyopic patients. Indeed, even if the published studies adopted training tasks as various as practicing Vernier acuity (Levi and Polat, 1996; Levi et al., 1997), position discrimination (Li and Levi, 2004; Li et al., 2005, 2007, 2008), contrast detection (Polat et al., 2004; Zhou et al., 2006), and letter identification (Levi, 2005; Chung et al., 2008), a certain degree of transfer to improvements in Snellen acuity globally emerges. This property is essential for amblyopia treatment, because the main deficit in amblyopia is reduced visual acuity and a substantial improvement in this basic visual function is required for a real advance of the patient quality of life.

It has been suggested that one reason why PL is so effective in reversing amblyopia in adult people might be that it requires subjects to make fine visual discriminations using their amblyopic eye under conditions of "active" visual system stimulation (Levi, 2005). Thus, visual attention may be a fundamental component of the therapeutic potential of PL. A recent study in non-amblyopic subjects provided indirect support to the important role of visual attention in driving visual cortex plasticity, showing that normalsighted people trained with action-based video games have robust improvements in basic visual functions (Li et al., 2009). The same effect was not observed after playing non-action video games that were equally engaging and visually complex, but operated at a slower pace and did not require precise visually guided actions. The effectiveness of this approach has promoted further research aimed at testing the value of active visual stimulation in amblyopic subjects. A substantial improvement in a wide range of visual functions including visual acuity, positional acuity, and stereopsis

\section{REFERENCES}

Bacci, A., Huguenard, J. R., and Prince, D. A. (2005). Modulation of neocortical interneurons: extrinsic influences and exercises in self-control. Trends Neurosci. 28, 602-610.

Baroncelli, L., Braschi, C., Spolidoro, M., Begenisic, T., Maffei, L., and Sale, A. (2011). Brain plasticity and disease: a matter of inhibition. Neural Plast. 2011, 286073-286083.

Baroncelli, L., Braschi, C., Spolidoro, M., Begenisic, T., Sale, A., and Maffei, L. (2010). Nurturing brain plasticity: impact of environmental enrichment. Cell Death Differ. 17, 1092-1103.

Bavelier, D., Levi, D. M., Li, R. W., Dan, Y., and Hensch, T. K. (2010). Removing brakes on adult brain plasticity: from molecular to behavioral interventions. J. Neurosci. 30, 14964-14971.

Berardi, N., Pizzorusso, T., and Maffei, L. (2000). Critical periods during sensory development. Curr. Opin. Neurobiol. 10, 138-145.

Brémond-Gignac, D., Copin, H., Lapillonne, A., and Milazzo, S. (2011). Visual development in infants: physiological and pathological mechanisms. Curr. Opin. Ophthalmol. 22, S1-S8.

Campbell, F. W., Hess, R. F., Watson, P. G., and Banks, R. (1978). Preliminary results of a physiologically based treatment of amblyopia. $\mathrm{Br}$. $\mathrm{J}$. Ophthalmol. 62, 748-755.

Chen, P. L., Chen, J. T., Fu, J. J., Chien, K. H., and Lu, D. W. (2008). A pilot study of anisometropic amblyopia improved in adults and children by perceptual learning: an alternative treatment to patching. Ophthalmic Physiol. Opt. 28, 422-428.

Chung, S. T. L., Li, R. W., and Levi, D. M. (2008). Learning to identify nearthreshold luminance-defined and contrast-defined letters in observers with amblyopia. Vision Res. 48, 2739-2750.

were also found in adults with amblyopia after a period of playing an action video game ( $\mathrm{Li}$ et al., 2011; Figure 1). In this case, vision recovery was also triggered by playing a non-action version of the games, leading to the interpretation that the threshold to elicit plasticity in a defective amblyopic visual eye might be lower than that required to achieve further improvement under conditions of normal vision (Bavelier et al., 2010).

As reviewed in the previous section, experiments made on rodent models of amblyopia have underscored a pivotal role of cortical GABAergic inhibition in limiting plasticity and amblyopia recovery in adulthood. Interestingly, the balance between excitation and inhibition has been suggested to be impaired during development also in amblyopic human subjects and cortical overinhibition could underlie the degradation of spatial vision abilities (Polat, 1999; Levi et al., 2002; Wong et al., 2005). In agreement with this hypothesis, repetitive transcranial magnetic stimulation (rTMS), which increases cortical excitability, transiently improves contrast sensitivity in adult amblyopes, likely acting on the excitation/inhibition balance (Thompson et al., 2008). A reduction of intracortical inhibition after rTMS has been also demonstrated in the motor cortex for both 1 and $10 \mathrm{~Hz}$ stimulations (Pascual-Leone et al., 1994; Modugno et al., 2003).

At the moment, it remains unknown whether the beneficial effects elicited by PL on amblyopia recovery are linked to changes in levels of brain inhibition. Preliminary experiments in our laboratory suggest a decrease of GABAergic inhibition in adult amblyopic rats that recover their visual functions in an active visual PL task. It is possible that the attention level required to perform PL tasks or to play video games might finally engage neuromodulatory systems of the brainstem, which may favor plasticity by increasing the excitatory/inhibitory ratio (Kasamatsu, 1991; Maya Vetencourt et al., 2008; Bavelier et al., 2010). Future studies should help further elucidate whether the molecular and cellular factors triggering brain plasticity in animal models are also crucial for a successful recovery of visual functions in human amblyopic subjects.

Cohen, I., Navarro, V., Clemenceau, S., Baulac, M., and Miles, R. (2002). On the origin of interictal activity in human temporal lobe epilepsy in vitro. Science 298, 1418-1421.

Cooke, S. F., and Bear, M. F. (2010). Visual experience induces longterm potentiation in the primary visual cortex. J. Neurosci. 30, 16304-16313.

Coull, J. A., Beggs, S., Boudreau, D., Boivin, D., Tsuda, M., Inoue, K., Gravel, C., Salter, M. W., and De Koninck, Y. (2005). BDNF from microglia causes the shift in neuronal anion gradient underlying neuropathic pain. Nature 438, 1017-1021.

Duffy, F. H., Burchfiel, J. L., and Conway, J. L. (1976). Bicuculline reversal of deprivation amblyopia in the cat. Nature 260, 256-257.

Fahle, M. (2004). Perceptual learning: a case for early selection. J. Vis. 4, 879-890.
Fahle, M. (2005). Learning to tell apples from oranges. Trends Cogn. Sci. (Regul. Ed.) 9, 455-457.

Fine, I., and Jacobs, R. A. (2002). Comparing perceptual learning tasks: a review. J. Vis. 2, 190-203.

Funk, K., Woitecki, A., Franjic-Würtz, C., Gensch, T., Möhrlen, F., and Frings, S. (2008). Modulation of chloride homeostasis by inflammatory mediators in dorsal root ganglion neurons. Mol. Pain 4, 32-44.

Gogolla, N., Leblanc, J. J., Quast, K. B., Südhof, T., Fagiolini, M., and Hensch, T. K. (2009). Common circuit defect of excitatory-inhibitory balance in mouse models of autism. $J$. Neurodev. Disord. 1, 172-181.

Harauzov, A., Spolidoro, M., DiCristo, G., De Pasquale, R., Cancedda, L., Pizzorusso, T., Viegi, A., Berardi, N., and Maffei, L. (2010). Reducing intracortical inhibition in the adult visual cortex promotes ocular dominance plasticity. J. Neurosci. 30, 361-371. 
He, H. Y., Ray, B., Dennis, K., and Quinlan, E. M. (2007). Experiencedependent recovery of vision following chronic deprivation amblyopia. Nat. Neurosci. 10, 1134-1136.

Hensch, T. K. (2005). Critical period plasticity in local cortical circuits. Nat. Rev. Neurosci. 6, 877-888.

Holmes, J. M., and Clarke, M. P. (2006). Amblyopia. Lancet 367, 1343-1351.

Huang, Z. J., Di Cristo, G., and Ango, F. (2007). Development of GABA innervation in the cerebral and cerebellar cortices. Nat. Rev. Neurosci. 8, 673-686.

Huberfeld, G., Wittner, L., Clemenceau, S., Baulac, M., Kaila, K., Miles, R., and Rivera, C. (2007). Perturbed chloride homeostasis and GABAergic signaling in human temporal lobe epilepsy. J. Neurosci. 27, 9866-9873.

Kasamatsu, T. (1982). Enhancement of neuronal plasticity by activating the norepinephrine system in the brain: a remedy for amblyopia. $\mathrm{Hum}$. Neurobiol. 1, 49-54.

Kasamatsu, T. (1991). Adrenergic regulation of visuocortical plasticity: a role of the locus coeruleus system. Prog. Brain Res. 88, 599-616.

Kiorpes, L. (2006). Visual processing in amblyopia: animal studies. Strabismus 14, 3-10.

Kirkwood, A., and Bear, M. F. (1994). Hebbian synapses in visual cortex. J. Neurosci. 14, 1634-1645.

Lagostena, L., Rosato-Siri, M., D’Onofrio, M., Brandi, R., Arisi, I., Capsoni, S., Franzot, J., Cattaneo, A., and Cherubini, E. (2010). In the adult hippocampus, chronic nerve growth factor deprivation shifts GABAergic signaling from the hyperpolarizing to the depolarizing direction. J. Neurosci. 30, 885-893.

Levi, D. M. (2005). Perceptual learning in adults with amblyopia: a re-evaluation of critical periods in human vision. Dev. Psychobiol. 46, 222-232.

Levi, D. M. (2006). Visual processing in amblyopia: human studies. Strabismus 14, 11-19.

Levi, D. M., Hariharan, S., and Klein, S. A. (2002). Suppressive and facilitatory spatial interactions in amblyopic vision. Vision Res. 42, 1379-1394.

Levi, D. M., and Li, R. W. (2009a). Perceptual learning as a potential treatment for amblyopia: a mini-review. Vision Res. 49, 2535-2549.

Levi, D. M., and Li, R. W. (2009b). Improving the performance of the amblyopic visual system. Philos. Trans. R. Soc. Lond. B Biol. Sci. 364, 399-407.
Levi, D. M., and Polat, U. (1996). Neural plasticity in adults with amblyopia. Proc. Natl. Acad. Sci. U.S.A. 93, 6830-6834.

Levi, D. M., Polat, U., and Hu, Y. S. (1997). Improvement in Vernier acuity in adults with amblyopia. Practice makes better. Invest. Ophthalmol. Vis. Sci. 38, 1493-1510.

Lewis, T. L., and Maurer, D. (2009). Effects of early pattern deprivation on visual development. Optom. Vis. Sci. 86, 640-646.

Li, R., Polat, U., Makous, W., and Bavelier, D. (2009). Enhancing the contrast sensitivity function through action video game training. Nat. Neurosci. 12, 549-551.

Li, R. W., Klein, S. A., and Levi, D. M. (2008). Prolonged perceptual learning of positional acuity in adult amblyopia: perceptual template retuning dynamics. J. Neurosci. 28, 14223-14229.

Li, R. W., and Levi, D. M. (2004). Characterizing the mechanisms of improvement for position discrimination in adult amblyopia. J. Vis. 4, 476-487.

Li, R. W., Ngo, C., Nguyen, J., and Levi, D. M. (2011). Video-game play induces plasticity in the visual system of adults with amblyopia. PLoS Biol. 9, e1001135. doi:10.1371/journal.pbio. 1001135

Li, R. W., Provost, A., and Levi, D. M. (2007). Extended perceptual learning results in substantial recovery of positional acuity and visual acuity in juvenile amblyopia. Invest. Ophthalmol. Vis. Sci. 48, 5046-5051.

Li, R. W., Young, K. G., Hoenig, P., and Levi, D. M. (2005). Perceptual learning improves visual perception in juvenile amblyopia. Invest. Ophthalmol. Vis. Sci. 46, 3161-3168.

Maya Vetencourt, J. F., Sale, A., Viegi, A., Baroncelli, L., De Pasquale, R., O'Leary, O. F., Castren, E., and Maffei, L. (2008). The antidepressant fluoxetine restores plasticity in the adult visual cortex. Science 320 , 385-388.

Mittelman, D. (2003). Amblyopia. Pediatr. Clin. North Am. 50, 189-196.

Modugno, N., Currà, A., Conte, A., Inghilleri, M., Fofi, L., Agostino, R., Manfredi, M., and Berardelli, A. (2003). Depressed intracortical inhibition after long trains of subthreshold repetitive magnetic stimuli at low frequency. Clin. Neurophysiol. 114, 2416-2422.

Morishita, H., Miwa, J. M., Heintz, N., and Hensch, T. K. (2010). Lynx1, a cholinergic brake, limits plasticity in adult visual cortex. Science 330, 1238-1240.
Pascual-Leone, A., Valls-Sole, J., Wassermann, E. M., and Hallett, M. (1994). Responses to rapid-rate transcranial magnetic stimulation of the human motor cortex. Brain 117, 847-858.

Pizzorusso, T., Medini, P., Landi, S. Baldini, S., Berardi, N., and Maffei, L. (2006). Structural and functional recovery from early monocular deprivation in adult rats. Proc. Natl. Acad. Sci. U.S.A. 103, 8517-8522.

Polat, U. (1999). Functional architecture of long-range perceptual interactions. Spat. Vis. 12, 143-162.

Polat, U., Ma-Naim, T., Belkin, M., and Sagi, D. (2004). Improving vision in adult amblyopia by perceptual learning. Proc. Natl. Acad. Sci. U.S. A. 101, 6692-6697.

Putignano, E., Lonetti, G., Cancedda, L. Ratto, G., Costa, M., Maffei, L., and Pizzorusso, T. (2007). Developmental downregulation of histone posttranslational modifications regulates visual cortical plasticity. Neuron 53 747-759.

Rozas, C., Frank, H., Heynen, A. J., Morales, B., Bear, M. F., and Kirkwood, A. (2001). Developmental inhibitory gate controls the relay of activity to the superficial layers of the visual cortex. J. Neurosci. 21, 6791-6801.

Sale, A., Berardi, N., and Maffei, L. (2009). Enrich the environment to empower the brain. Trends Neurosci. 32, 233-239.

Sale, A., De Pasquale, R., Bonaccorsi, J. Pietra, G., Olivieri, D., Berardi, N., and Maffei, L. (2011). Visual perceptual learning induces long-term potentiation in the visual cortex. Neuroscience 172, 219-225.

Sale, A., Maya Vetencourt, J. F., Medini, P., Cenni, M. C., Baroncelli, L., De Pasquale, R., and Maffei, L. (2007). Environmental enrichment in adulthood promotes amblyopia recovery through a reduction of intracortical inhibition. Nat. Neurosci. 10 679-681.

Silingardi, D., Scali, M., Belluomini, G. and Pizzorusso, T. (2010). Epigenetic treatments of adult rats promote recovery from visual acuity deficits induced by long-term monocular deprivation. Eur. J. Neurosci. 31, 2185-2192.

Smith, G. B., and Bear, M. F. (2010). Bidirectional ocular dominance plasticity of inhibitory networks: recent advances and unresolved questions. Front Cell. Neurosci. 4:21 doi:10.3389/fncel.2010.00021

Spolidoro, M., Baroncelli, L., Putignano, E., Maya Vetencourt, J. F., Viegi, A., and Maffei, L. (2011). Food restriction enhances visual cortex plasticity in adulthood. Nat. Commun. 2, 320-327.
Stewart, C. E., Fielder, A. R., Stephens, D. A., and Moseley, M. J. (2005). Treatment of unilateral amblyopia: factors influencing visual outcome. Invest. Ophthalmol. Vis. Sci. 46, 3152-3160.

Thompson, B., Mansouri, B., Koski, L., and Hess, R. F. (2008). Brain plasticity in the adult: modulation of function in amblyopia with rTMS. Curr. Biol. 18, 1067-1071.

van Praag, H., Kempermann, G., and Gage, F. H. (2000). Neural consequences of environmental enrichment. Nat. Rev. Neurosci. 1, 191-198.

Weliky, M. (2000). Correlated neuronal activity and visual cortical development. Neuron 27, 427-430.

Wiesel, T. N., and Hubel, D. H. (1963). Single-cell responses in striate cortex of kittens deprived of vision in one eye. J. Neurophysiol. 26, 1003-1017.

Wong, E. H., Levi, D. M., and McGraw, P. V. (2005). Spatial interactions reveal inhibitory cortical networks in human amblyopia. Vision Res. 45, 2810-2819.

Wu, C., and Hunter, D. G. (2006). Amblyopia: diagnostic and therapeutic options. Am. J. Ophthalmol. 141, 175-184.

Yotsumoto, Y., Watanabe, T., and Sasaki, Y. (2008). Different dynamics of performance and brain activation in the time course of perceptual learning. Neuron 57, 827-833.

Zhou, Y., Huang, C., Xu, P., Tao, L., Qiu, Z., Li, X., and Lu, Z. (2006). Perceptual learning improves contrast sensitivity and visual acuity in adults with anisometropic amblyopia. Vision Res. 46, 739-750.

Conflict of Interest Statement: The authors declare that the research was conducted in the absence of any commercial or financial relationships that could be construed as a potential conflict of interest.

Received: 26 September 2011; paper pending published: 11 October 2011; accepted: 07 November 2011; published online: 24 November 2011.

Citation: Baroncelli L, Maffei L and Sale A (2011) New perspectives in amblyopia therapy on adults: a critical role for the excitatory/inhibitory balance. Front. Cell. Neurosci. 5:25. doi: 10.3389/fncel.2011.00025

Copyright (C) 2011 Baroncelli, Maffei and Sale. This is an open-access article subject to a non-exclusive license between the authors and Frontiers Media SA, which permits use, distribution and reproduction in other forums, provided the original authors and source are credited and other Frontiers conditions are complied with. 